Session 2268

\title{
The Role of the Kinetic Diagram in the Teaching of Introductory Rigid-Body Dynamics - Past, Present, and Future
}

\author{
Glenn Kraige \\ Department of Engineering Science and Mechanics \\ Virginia Polytechnic Institute and State University \\ Blacksburg, VA 24061 USA
}

\begin{abstract}
The introductory engineering dynamics course is widely regarded as one of the most difficult courses that the undergraduate engineering student takes. Further, the rigid-body area of this dynamics course is considered much more difficult than the particle area. One reason for the latter statement is that we have not yet progressed to the best steady-state teaching strategy in the area of rigid-body kinetics. The purpose of this paper is to review the history and current state of affairs in this narrow area and then to advocate a better strategy. Recommendations are made in regard to both diagrams and corresponding equations of motion.
\end{abstract}

\section{Introduction}

Dynamics did not become a significant issue until the beginning of the machine age. Mechanicists were accustomed to a zero on the right-hand side of the governing equations in statics, so the first direction of particle dynamics was to include a - $m a$ term on the left side of dynamics equations so that the right-hand zero could be retained. Although sometimes referred to as D'Alembert's Principle, this technique should be called dynamic equilibrium (D'Alembert's Principle is a virtual-work principle). This - $m a$ term has been called an inertia force, an effective force, a reversed effective force, etc. The technical community eventually took the position that dynamics should not be treated as a special case of statics, but rather the other way around. In other words, we soon placed the ma term on the right side of the equations of motion and included only real (contact and body) forces on the left side. Some textbooks went through a period in which a kinetic diagram (sometimes called a resultant-force diagram) was drawn (in addition to the free-body diagram (FBD)). This diagram merely showed an $m a$ vector (or its components). The usual arrangement was to draw the FBD and then write an equal sign with the kinetic diagram (KD) to the right. Such practice seems to have been largely terminated for particle dynamics.

It is the author's position that, with the conventional teaching of particle dynamics as outlined above, we are in the steady-state, terminal teaching configuration. We note that the teaching of particle statics and particle dynamics is now of identical format. There is the same type of FBD showing only real forces, followed by application of governing equations. The only difference is that, for particle dynamics, the right-hand sides of the governing equations are not zero. 
The story is not yet complete in rigid-body dynamics, however. Again, moving back to the dawn of the machine age, the first attempts at planar rigid-body dynamics were based upon the familiar customs of statics: $-m \bar{a}$ ( $\bar{a}$ refers to the mass center here) and $-\bar{I} \alpha$ vectors $(\bar{I}$ refers to the mass center) were placed directly on the FBD so that they could be treated as an effective force and couple, allowing the use of statics-like equations of motion. As was the case in statics, the educational community eventually rebelled against considering the $-m \bar{a}$ and $-\bar{I} \alpha$ vectors to be the same as real forces and moments in association with the principle of dynamic equilibrium. Rather than eliminating these quantities completely from diagrammed material, however, they were moved to a second diagram (the kinetic diagram) as positive quantities - in effect, they were moved from the left to the right side of the governing equation for rotational motion. The reason this second diagram became "necessary" had to do with the fact that the $m \bar{a}$ vector might enter the moment equation (depending on what point was chosen as the moment center).

Most of the popular introductory dynamics texts ${ }^{1-8}$ use the kinetic-diagram approach outlined above, but only for rigid-body kinetics and not for particle kinetics. Thus, at present, the mainstream teaching strategy consists of one approach for particle and rigid-body statics and particle kinetics, but a different approach (in terms of diagrams) for rigid-body kinetics.

The third approach to rigid-body kinetics is to use only a FBD and appropriate governing equations, so that all categories of statics and dynamics are treated in parallel fashions. This philosophy, which was recommended by the author in $1982^{9}$, will be expanded in the sections that follow.

\section{Discussion of the Three Methods}

In Figure 1, we show generic diagrams associated with the three approaches introduced above, as applied to an arbitrary two-dimensional slab. In the dynamic-equilibrium approach of Figure 1a, we show the applied forces along with $-m \bar{a}$ and $-\bar{I} \alpha$ vectors. The governing equations associated with this approach are

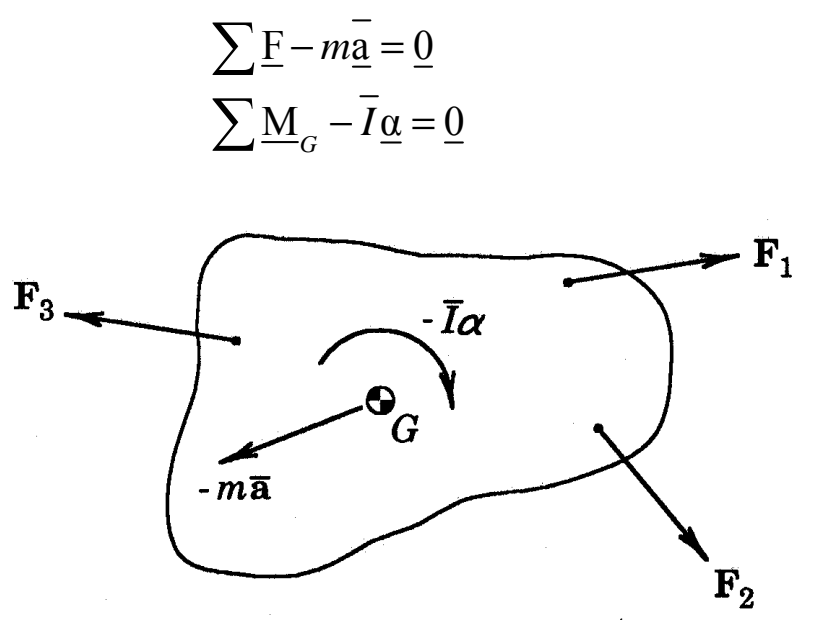

Figure 1a: The Dynamic-Equilibrium Approach 
One of the disadvantages associated with this method, in addition to treating the kinetic results ( $m \bar{a}$ and $\bar{I} \alpha$ ) the same as if they were causing agents (the applied forces and couples), is that one must decide on a strategy for assuming the senses of $\bar{a}$ and $\alpha$. Does one anticipate the correct sense of these two quantities (and then reverse them!), or does one always assume that they are in the positive coordinate directions?

In Figure 1b, we show a conventional FBD on the left and on the right is the kinetic diagram indicating the $m \bar{a}$ and $\bar{I} \alpha$ vectors. The governing equations associated with this approach are generally written as

$$
\begin{aligned}
\sum \underline{\mathrm{F}} & =m \underline{\overline{\mathrm{a}}} \\
\sum M_{P} & =\bar{I} \alpha+m \bar{a} d \quad\left(\text { or } \sum \underline{\mathrm{M}}_{P}=\bar{I} \underline{\alpha}+\underline{\rho} \times m \underline{\overline{\mathrm{a}}}\right)
\end{aligned}
$$
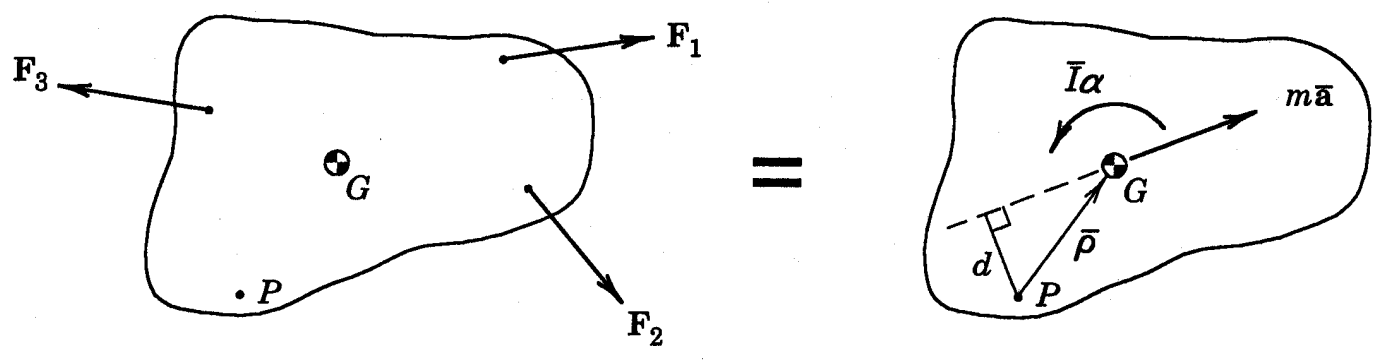

Figure 1b: The Kinetic-Diagram Approach

The governing rotational equation can be derived in at least two ways. First, and best, one can develop it from first principles. This may be done by considering a general three-dimensional system of particles or by considering a rigid slab as is done in Appendix A. A second derivation is to perform a moment sum about any point in the FBD and equate the results to the moment sum about the same point in the kinetic diagram (KD). The argument is that because the two diagrams are equivalent, performing the same operations in the respective diagrams must also be equivalent. The author finds this second derivation somewhat lacking. But, in fairness, it must be noted that the introductory course does usually not allow sufficient time for the first derivation. And the first derivation does require more effort on the part of the student.

An often-cited advantage of the KD approach is the ability to sum moments about a convenient point - that is, a point through which several unknown forces might pass. As we will note below, this advantage is not confined to the KD approach. Furthermore, the method involves the acceleration of the mass center $G$, which may not be known.

As a final comment on the kinetic-diagram method, we note that its use in three-dimensional problems becomes quite labored. A good question for all mechanics educators is "If we do not use the KD for 3-D problems, then why do we introduce it for 2-D problems?" 
In Figure 1c is the pure FBD approach. It is clear that we can always sum moments about the mass center $G$ or about a fixed point $O$ on the body (if one exists). We list as the equation of rotational motion Eq. (A/9) of Appendix A, in order that we might cite its advantages. The author believes that this form is much more powerful than Eq. (A/8), because it gives one the ability to conveniently sum moments about a point whose acceleration is known, which is a frequent occurrence. It of course reduces to the familiar forms if $P$ is the mass center or if $P$ is a nonaccelerating point fixed to the body.

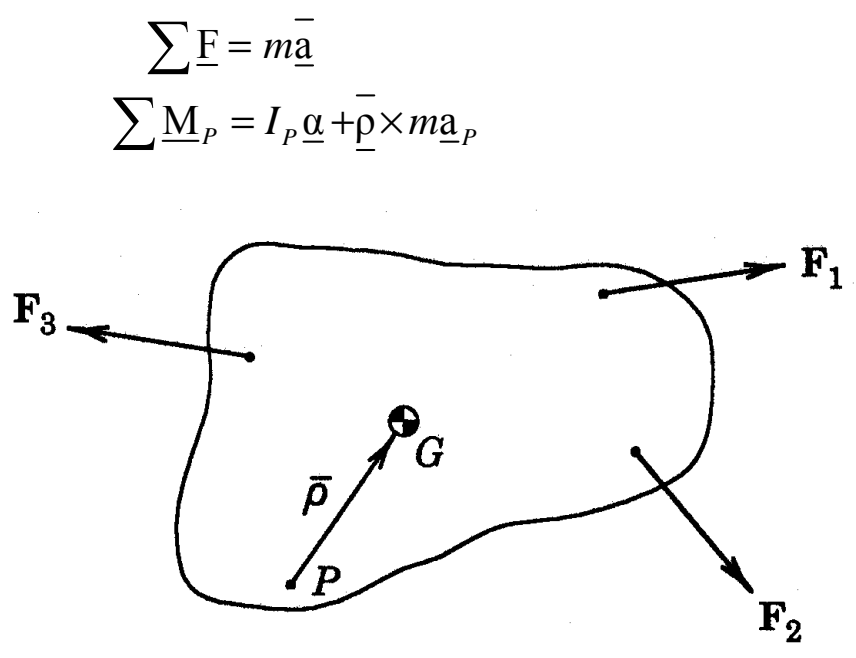

Figure 1c: The Pure FBD Approach

We now turn to a specific problem: Suppose the 3000-lb car is brought to a halt by skidding all tires. If the coefficient of kinetic friction is 0.8 , determine the normal reaction force under the pair of front wheels, that under the pair of rear wheels, and the deceleration during the braking period.

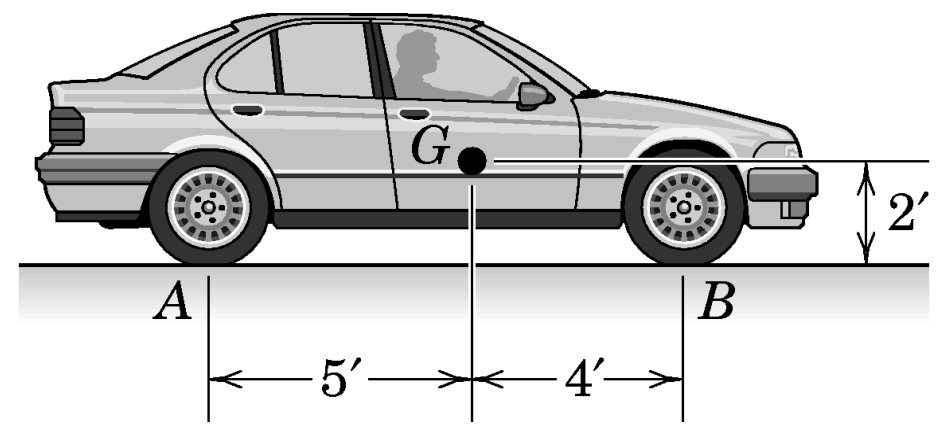

Figure 2: A Sample Problem

Three solutions to the problem are indicated below. 
I. Dynamic-Equilibrium Equations of Motion

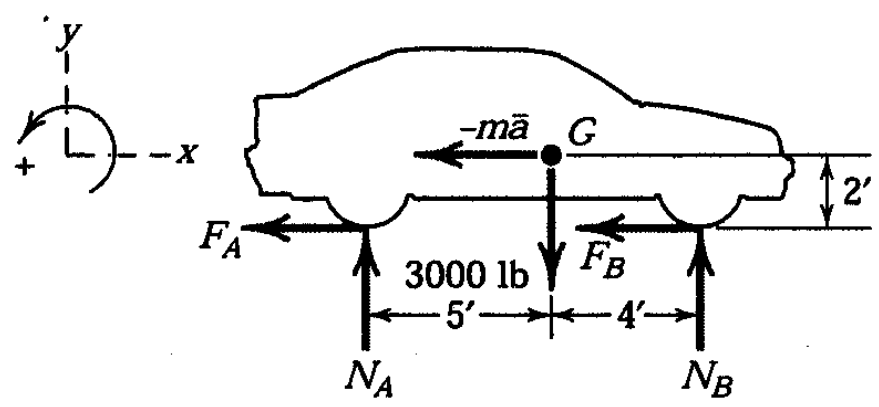

Figure 2a

$$
\begin{array}{ll}
\sum F_{x}=0: & -0.8 N_{A}-0.8 N_{B}-\frac{3000}{32.2} \bar{a}=0 \\
\sum F_{y}=0: & N_{A}+N_{B}-3000=0 \\
\sum M_{A}=0: & -5(3000)+9 N_{B}+\frac{3000}{32.2} \bar{a}(2)=0
\end{array}
$$

(Point $A$ is chosen so as to reduce the number of unknowns in the rotational equation.)

II. Kinetic-Diagram Equations of Motion

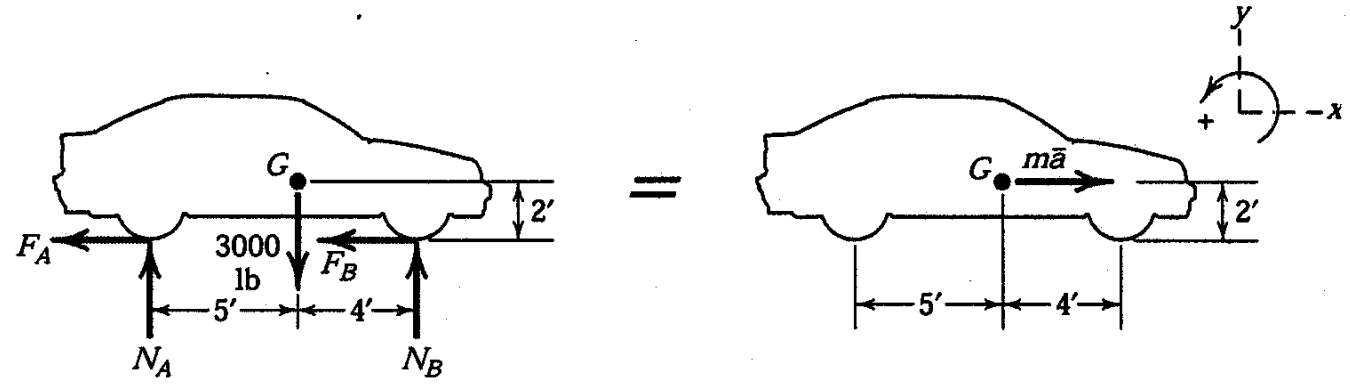

Figure $2 b$

$$
\begin{array}{ll}
\sum F_{x}=m \bar{a}_{x}: & -0.8 N_{A}-0.8 N_{B}=\frac{3000}{32.2} \bar{a} \\
\sum F_{y}=m \bar{a}_{y}: & N_{A}+N_{B}-3000=0 \\
\sum M_{A}=\bar{I} \alpha+m \bar{a} d: & -5(3000)+9 N_{B}=0-\frac{3000}{32.2} \bar{a}(2)
\end{array}
$$

(Point $A$ is chosen so as to reduce the number of unknowns in the rotational equation.) 
III. Pure FBD Equations of Motion

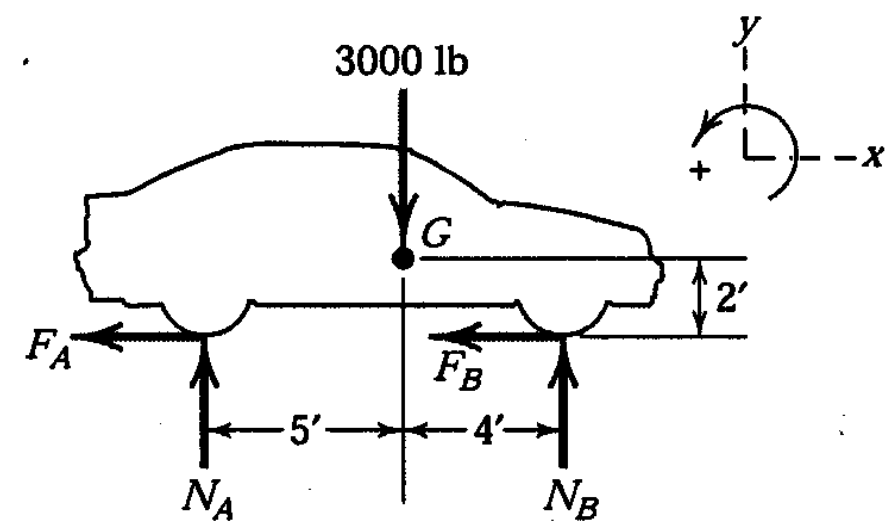

Figure 2c

$$
\begin{aligned}
& \sum F_{x}=m \bar{a}_{x}: \quad-0.8 N_{A}-0.8 N_{B}=\frac{3000}{32.2} \bar{a} \\
& \sum F_{y}=m \bar{a}_{y}: \quad N_{A}+N_{B}-3000=0 \\
& \sum M_{G}=\bar{I} \alpha: \quad-2\left(0.8 N_{A}+0.8 N_{B}\right)-5 N_{A}+4 N_{B}=0
\end{aligned}
$$

As an alternative to the choice of $G$ as a moment center, we could choose point $A$ :

$$
\sum \underline{\mathrm{M}}_{A}=I_{A} \underline{\alpha}+\underline{\bar{\rho}} \times m \underline{\mathrm{a}}_{A}:-5(3000)+9 N_{B}=0-2\left(\frac{3000}{32.2}\right) \bar{a}
$$

This is the same as the rotational equation for the kinetic diagram approach.

This sample problem is chosen because it was part of a 1982 poll $^{9}$ of 73 faculty members. The results of that poll are shown in the table below. The percentages shown indicate the degree of support for each of the three solutions above.

\begin{tabular}{|l|c|c|}
\hline & All Faculty & Dynamics Faculty \\
\hline $\begin{array}{l}\text { Solution I } \\
\text { (Dynamic Equilibrium) }\end{array}$ & $6.8 \%$ & $2.6 \%$ \\
\hline $\begin{array}{l}\text { Solution II } \\
\text { (Kinetic Diagram) }\end{array}$ & $17.1 \%$ & $7.9 \%$ \\
\hline $\begin{array}{l}\text { Solution III } \\
\text { (Pure FBD) }\end{array}$ & $76.0 \%$ & $89.5 \%$ \\
\hline
\end{tabular}

The pure FBD method was the clear choice of all faculty polled; an overwhelming major of those who list dynamics as their specialty prefer this method.

As a final example, we look to a typical rigid-body vibrations problem: Determine the equation of small motion for the pendulum whose pivot point $P$ is subjected to the harmonic displacement $x_{P}=b \cos \omega t$. 

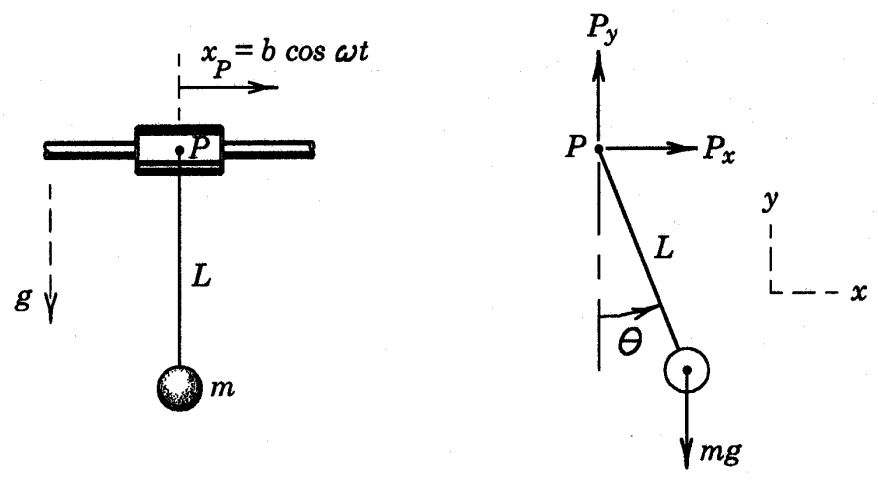

Figure 3

The solution begins with the FBD shown. We choose the accelerating pivot point $P$ as the moment center.

$$
\begin{aligned}
& \sum \underline{\mathrm{M}}_{P}=I_{P} \underline{\underline{\alpha}}+\underline{-} \underline{\underline{\rho}} \times m \underline{\mathrm{a}}_{P}: \\
& -m g L \sin \theta \underline{\mathrm{k}}=m L^{2} \ddot{\theta} \underline{\mathrm{k}}+L(\sin \theta \underline{\mathrm{i}}-\cos \theta \underline{\mathrm{j}}) \times m\left(-b \omega^{2} \cos \omega t\right) \underline{\mathrm{i}}
\end{aligned}
$$

Simplifying for small $\theta$ and equating coefficients of $\underline{\mathrm{k}}$ yield the equation of motion

$$
\ddot{\theta}+\frac{g}{L} \theta=\frac{b}{L} \omega^{2} \cos \omega t
$$

We see that the pure free-body diagram method is extremely efficient for such problems.

\section{Conclusions and Recommendations}

The pure FBD method is hereby recommended for solving all rigid-body kinetics problems. Such consistent instruction should begin in the introductory dynamics course and be carried through all subsequent dynamics and vibrations courses. The following reasons support this recommendation:

1. The student uses the same diagram for all types of mechanics problems - statics and dynamics, particle and rigid-body. Thus, use of the FBD approach would give a unity and simplicity to mechanics education.

2. The necessary equation-of-motion developments are understandable by the average sophomore engineering student.

3. The method involves no fictitious force or fictitious moment vectors.

4. Fewer decisions (as to which direction acceleration vectors should have) are required. 
5. The student is better able to see that the equation of rotational motion referenced to a mass center or a fixed point is a special case of the equation referenced to an arbitrary point.

6. The method is as good as any other from the viewpoint of choosing convenient moment centers.

7. At least one poll suggests that the majority of instructors, especially those whose specialty is dynamics, prefer this approach.

\section{Acknowledgments}

The illustrations contained in this paper were drawn by J. C. Morgan and Tom Brucker of Precision Graphics and by David Kraige. The editorial suggestions and historical contributions of Dr. Albert L. Hale are duly recognized. Finally, the word-processing contributions of Vanessa McCoy are gratefully acknowledged.

\section{References}

1. Bedford, A. and Fowler, W., Engineering Mechanics: Dynamics, Addison Wesley, 1999.

2. Beer, F. P. and Johnston, E. R., Jr., Vector Mechanics for Engineers - Statics and Dynamics, Sixth Edition, McGraw-Hill Book Co., 1997.

3. Hibbeler, R. C., Engineering Mechanics - Dynamics, Eighth Edition, Prentice-Hall, Inc., 1998.

4. Meriam, J. L. and Kraige, L. G., Engineering Mechanics: Dynamics, Fifth Edition, John Wiley and Sons, Inc., 2002.

5. Pytel, A. and Kiusalaas, J., Engineering Mechanics: Dynamics, Second Edition, Brooks/Cole Publishing Co., 1999.

6. Riley, W. F. and Sturges, L. D., Engineering Mechanics: Dynamics, John Wiley and Sons, Inc., 1996.

7. Shames, I. H., Engineering Mechanics: Dynamics, Third Edition, Prentice-Hall, Inc., 1980.

8. Soutas-Little, R. W. and Inman, D. J., Engineering Mechanics: Dynamics, Prentice-Hall, 1999.

9. Kraige, L. G., "The Teaching of Planar Rigid-Body Dynamics to Sophomore Engineering Students", 1982 ASEE Annual Conference Proceedings, pp. 750-758.

10. Hale, A. L., Private communication, February 2002.

\section{Appendix A}

Contained in this Appendix are fairly standard derivations for key equations of rotational motion for rigid bodies. Clearly, there is nothing new here; the material is presented for the sole purpose of stressing its suitability for use in an introductory course.

Consider the two-dimensional rigid body of Figure A/1. Point $G$ is the mass center, and point $P$ is an arbitrary body-fixed point. The vectors $\underline{\rho}_{i}, \underline{\rho}_{i}^{\prime}$, and $\underline{\rho}$ are position vectors. The mass of the element is $m_{i}$, and all summations below are understood to be taken over all such elements of the rigid slab. 


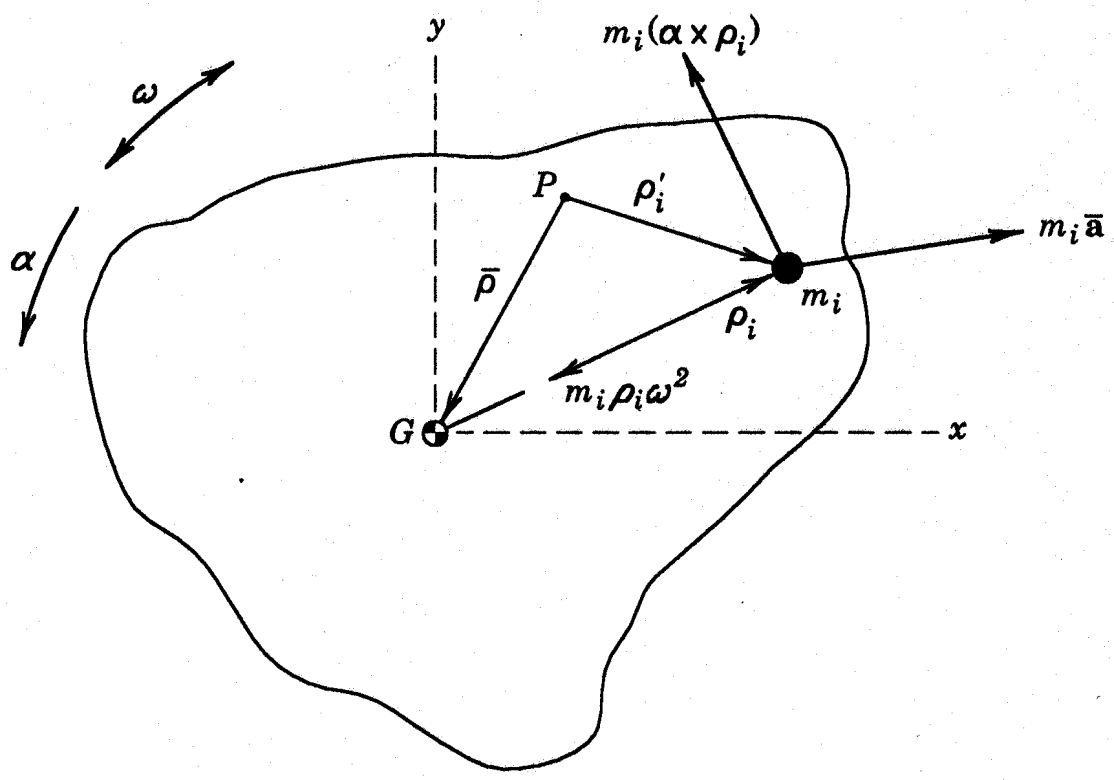

Figure A/1

The resultant force acting on the element of mass $m_{i}$ is

$$
\sum \underline{\mathrm{F}}_{i}=m_{i} \underline{\mathrm{a}}_{i}=m_{i}\left(\underline{\overline{\mathrm{a}}}+\underline{\alpha} \times \underline{\rho}_{i}-\omega^{2} \underline{\rho}_{i}\right)
$$

where $\underline{\alpha}$ and $\underline{\omega}$ are the angular acceleration and angular velocity, respectively, of the body.

\section{The mass center $G$ as a reference point}

The moment about $G$ of the resultant force acting on the element of mass $m_{i}$ is

$$
\underline{\mathrm{M}}_{G_{i}}=\underline{\rho}_{i} \times m_{i} \underline{\mathrm{a}}_{i}=\underline{\rho}_{i} \times m_{i}\left(\underline{\alpha} \times \underline{\rho}_{i}\right)+\underline{\rho}_{i} \times m_{i} \overline{\mathbf{a}}
$$

The sum of all such moments is

$$
\begin{aligned}
\underline{\mathrm{M}}_{G} & =\sum \underline{\mathrm{M}}_{G_{i}}=\sum \underline{\rho}_{i} \times m_{i}\left(\underline{\alpha} \times \underline{\rho}_{i}\right)+\sum \underline{\rho}_{i} \times m_{i} \overline{\overline{\mathrm{a}}} \\
& =\sum m_{i} \rho_{i}^{2} \underline{\alpha}+\sum m_{i} \underline{\rho}_{i} \times \underline{\mathrm{a}}
\end{aligned}
$$

or

$$
\underline{\mathrm{M}}_{G}=\bar{I} \underline{\alpha}
$$

\section{A fixed point $O$ as a reference point}

If a fixed point $O$, rather than the mass center $G$, is taken as the reference point and origin of coordinates in Fig. A/1, we obtain 


$$
\underline{\mathrm{M}}_{O}=I_{O} \underline{\alpha}
$$

because $\bar{a}$ in the above derivation is replaced by $\underline{\mathrm{a}}_{o}=\underline{0}$.

\section{An arbitrary point $P$ as a reference point}

The moment about the arbitrary point $P$ of the resultant force acting on the element of mass $m_{i}$ is

$$
\underline{\mathrm{M}}_{\underline{P}_{i}}=\underline{\rho}_{i}^{\prime} \times m_{i} \underline{\mathrm{a}}_{i}=\underline{\rho}_{i}^{\prime} \times m_{i}\left(\underline{\bar{a}}+\underline{\alpha} \times \underline{\rho}_{i}-\omega^{2} \underline{\rho}_{i}\right)
$$

The sum of all such moments is

$$
\underline{\mathrm{M}}_{P}=\sum \underline{\mathrm{M}}_{P_{i}}=\sum\left[\underline{\rho}_{i}^{\prime} \times m_{i}\left(\underline{\bar{a}}+\underline{\alpha} \times \underline{\rho}_{i}-\omega^{2} \underline{\rho}_{i}\right)\right]
$$

Recognizing that $\underline{\rho}_{i}^{\prime}=\underline{\bar{\rho}}+\underline{\rho}_{i}$, we write the first term of Eq. A/4 as

$$
\begin{aligned}
T_{1} & =\sum\left(\bar{\rho}+\underline{\rho}_{i}\right) \times m_{i} \underline{\overline{\mathrm{a}}} \\
& =\sum \overline{\underline{\rho}} \times m_{i} \overline{\mathrm{a}}+\sum \underline{\rho}_{i} \times m_{i} \overline{\mathrm{a}} \\
& =\bar{\rho} \times \overline{\bar{\rho}} \sum m_{i}+\sum m_{i} \rho_{i} \times \underline{\overline{\mathbf{a}}} \\
& =\underline{\bar{\rho}} \times m_{\overline{\mathbf{a}}}
\end{aligned}
$$

where the definition of the mass center $G$ has been utilized.

The second term of Eq. A/4 is

$$
\begin{aligned}
T_{2} & =\sum\left(\underline{\bar{\rho}}+\underline{\rho}_{i}\right) \times m_{i}\left(\underline{\alpha} \times \underline{\rho}_{i}\right) \\
& =\sum \underline{\underline{\rho}} \times m_{i}\left(\underline{\alpha} \times \underline{\rho}_{i}\right)+\sum \underline{\rho}_{i} \times m_{i}\left(\underline{\alpha} \times \underline{\rho}_{i}\right) \\
& =\bar{\rho} \times\left(\underline{\alpha} \times \sum m_{i} \underline{\rho}_{i}\right)+\sum m_{i} \rho_{i}^{2} \underline{\alpha} \\
& =\bar{I} \underline{\alpha}
\end{aligned}
$$

The third term of Eq. $\mathrm{A} / 4$ is

$$
\begin{aligned}
T_{3} & =\sum\left(\underline{\bar{\rho}} \times \underline{\rho}_{i}\right) \times m_{i}\left(-\omega^{2} \underline{\rho}_{i}\right) \\
& =\sum \underline{\bar{\rho}} \times \omega^{2} m_{i} \underline{\rho}_{i}-\sum \underline{\rho}_{i} \times \omega^{2} m_{i} \underline{\rho}_{i} \\
& =\underline{\bar{\rho}} \times \omega^{2} \sum m_{i} \underline{\rho}_{i} \\
& =\underline{0}
\end{aligned}
$$


Again, the definition of the mass center has been used. So Eq. (A/4) simplifies to

$$
\underline{\mathrm{M}}_{P}=\bar{I} \underline{\alpha}+\underline{\bar{\rho}} \times m \underline{\mathbf{a}}
$$

Because $\underline{\overline{\mathrm{a}}}=\underline{\mathrm{a}}_{G}=\underline{\mathrm{a}}_{P}+\underline{\mathrm{a}}_{G / P}$, we can write Eq. (A/8) as

$$
\begin{aligned}
\underline{\mathrm{M}}_{P} & =\bar{I} \underline{\alpha}+\underline{\bar{\rho}} \times m\left(\underline{\mathrm{a}}_{P}+\underline{\alpha} \times \underline{\bar{\rho}}-\omega^{2} \bar{\rho}\right) \\
& =\bar{I} \underline{\alpha}+\underline{\bar{\rho}} \times m \underline{\mathrm{a}}_{P}+m \underline{\underline{\rho}} \times(\underline{\alpha} \times \underline{\bar{\rho}})-m \omega^{2} \underline{\underline{\rho}} \times \underline{\rho} \\
& =\bar{I} \underline{\alpha}+m \bar{\rho} \underline{\alpha}+\underline{\underline{\rho}} \times m \underline{\mathrm{a}}_{P}
\end{aligned}
$$

or

$$
\underline{\mathrm{M}}_{P}=I_{P} \underline{\alpha}+\underline{\bar{\rho}} \times m \underline{\mathrm{a}}_{P}
$$

\section{GLENN KRAIGE}

Currently Professor of Engineering Science and Mechanics at Virginia Tech, Glenn Kraige has 27 years of teaching experience. His professional interests include general dynamics, spacecraft attitude dynamics and control, vibrations, and engineering education. His B.S., M.S., and Ph.D. are from The University of Virginia. 